\title{
Acetazolamide as Add-on Diuretic Therapy in Exacerbations of Chronic Heart Failure: a Pilot Study
}

\author{
Tomasz Imiela ${ }^{1}$ (D) Andrzej Budaj ${ }^{1}$
}

Published online: 30 September 2017

(c) The Author(s) 2017. This article is an open access publication

\begin{abstract}
Background Congestion is the main cause of morbidity in patients with heart failure. Treatment of fluid overload is often challenging in everyday clinical practice.

Objective The aim of this study was to determine the diuretic effect of acetazolamide in patients with exacerbations of chronic heart failure, in addition to their stable diuretic therapy.

Methods This was a single-center, unblinded study. Patients hospitalized with chronic heart failure exacerbations, with left ventricular ejection fraction $(\mathrm{EF})<50 \%$ and signs of volume overload, with a stable dose of diuretics anticipated by the attending physician over the next 4 days, were considered eligible for the study. On day 1 , patients were randomized to receive acetazolamide orally, once daily (dose-adjusted to body weight) or no treatment (control group) as add-on diuretic therapy, on days 2 and 3. Diuresis, natriuresis, fluid balance, and symptoms were assessed daily, up to day 4 .

Results Twenty patients (mean \pm standard deviation age $72 \pm 11.6$ years; $85 \%$ men; mean EF $33.8 \pm 11.4 \%$; mean $\mathrm{N}$-terminal pro-B-type natriuretic peptide $8064 \pm 5593 \mathrm{pg} / \mathrm{mL}$; mean intravenous furosemide dose $105 \pm 55 \mathrm{mg}$ ) were enrolled. Diuresis, natriuresis, fluid balance, and symptoms were stable on days 1-4 in the control group. An increase in diuresis and natriuresis, and a greater change in fluid balance after administration of acetazolamide, were observed in patients randomized to acetazolamide. On day 4 , there was a significant difference
\end{abstract}

Tomasz Imiela

tomasz.imiela1@gmail.com

1 Cardiology Department, Postgraduate Medical School, Grenadierow 51/59, 04-073 Warsaw, Poland in fluid balance between the acetazolamide and control groups $\quad(-666 \pm 1194 \mathrm{~mL} \quad$ vs. $\quad+332 \pm 705 \mathrm{~mL}$; $p=0.035)$, and dyspnea was lower in patients receiving acetazolamide (visual scale, $p<0.001$; 5-point Likert scale, 1.444 vs. $2.222 ; p=0.04$ )

Conclusions In this pilot study, the addition of acetazolamide to the background diuretic regimen in patients with chronic heart failure exacerbations produced an additional diuretic effect and alleviation of dyspnea.

\section{Key Points}

Acetazolamide, in addition to other diuretics, may improve diuresis in heart failure exacerbations

Acetazolamide may decrease dyspnea in heart failure exacerbations

\section{Introduction}

Heart failure is a common disease associated with a high risk of hospitalization and premature death. The prevalence of heart failure is approximately $1-2 \%$ of the population in developed countries, with the risk of developing the disease rising with age [1].

Fluid retention is the cause of numerous symptoms in chronic heart failure [2]. The basic method for reducing fluid retention in heart failure is the administration of diuretics, but there is no evidence that this approach decreases the fatality rate. Nevertheless, guidelines from 
the European Society of Cardiology (ESC) recommend administration of diuretics, both in the acute and chronic phases, to obtain proper fluid balance in patients with overhydration symptoms [3].

Historically, one of the first classes of diuretics used to achieve decongestion in heart failure were the carbonic anhydrase inhibitors. The currently available drug from that class, acetazolamide, acts through blockade of carbonic anhydrase in various organs. In the kidney, carbonic anhydrase is expressed mainly in proximal tubules. With its blockade, acetazolamide increases natrium and bicarbonate excretion, urine alkalosis, and blood acidosis [4]. Anhydrase inhibitors were used in the treatment of volume overload in heart failure, in combination with mercurial diuretics, in the 1950s and 1960s [5]. With the discovery of potent loop diuretics, the use of anhydrase inhibitors has been largely abandoned (at least in the treatment of heart failure with volume overload).

Diuretic resistance is a growing clinical problem in heart failure, and patients resistant to loop diuretics have a poor prognosis [6]. Anhydrase inhibitors, which are currently used occasionally in such patients, have not been tested in the era of evidence-based medicine; however, limited animal and human data suggest a potential benefit of acetazolamide, especially in combination with loop diuretics, in the treatment of fluid overload in heart failure [7]. Consequently, we conducted a pilot study to determine the putative clinical value of adding acetazolamide on top of the current diuretic therapy in patients with heart failure and signs of volume overload.

\section{Patients and Methods}

\subsection{Study Design}

This prospective, randomized, unblinded, single-center study was carried out in the Cardiology Department of the Centre of Postgraduate Medical Education, Warsaw, Poland, between June 2015 and February 2016. All patients provided written informed consent and the study was approved by the institutional Ethics Committee.

\subsection{Population}

Consecutive patients admitted to the cardiology ward with exacerbations of acute or chronic heart failure were screened. Eligible patients met the following criteria: $\mathrm{x}$-ray-confirmed pulmonary congestion and/or the presence of signs of excess fluid (leg edema, ascites) requiring hospitalization (only patients with persistent signs of fluid overload during the initial evaluation were included); left ventricular ejection fraction $(\mathrm{EF})<50 \%$; stable diuretic therapy, defined as a stable dose of diuretics anticipated by the attending physician over the next 4 days; age $\geq 18$ years; and the possibility of precise estimation of daily diuresis, determined by the investigator (i.e. patients with a Foley catheter inserted in the urinary bladder, or noncatheterized patients for whom reliable fluid balance control had been observed for the previous days).

Exclusion criteria were current acute coronary syndrome, acute pulmonary embolism, acute myocarditis, aortic dissection, stage 5 chronic kidney disease (estimated glomerular filtration rate $<15 \mathrm{~mL} / \mathrm{min}$ ) or current hypotension (mean arterial pressure $<60 \mathrm{mmHg}$ ).

\subsection{Treatment Protocol}

The study design is illustrated in Fig. 1. Patients were observed over 4 consecutive days. On day 1, patients were randomized to receive acetazolamide orally, once daily, with dose-adjustment for body weight $(<75 \mathrm{~kg}, 250 \mathrm{mg}$; $75-100 \mathrm{~kg}, 375 \mathrm{mg} ;>100 \mathrm{~kg}, 500 \mathrm{mg}$ ), or no additional treatment (control group). To measure the fluid output on diuretics used before the initiation of acetazolamide and compare it with the consecutive days with the addition of a study drug, acetazolamide was only administered on days 2 and 3. Randomization was performed using a computer-generated 16-bit exact randomization procedure performed prior to commencement of the study. Treating physicians were discouraged from changing the dose of background diuretics unless considered necessary per the patient's clinical condition. Detailed data on the dose of diuretics and other medications were collected throughout the study.

\subsection{Laboratory Measurements}

Daily natrium and chloride urine excretion were measured on all 4 study days. On days 1 and 4, serum natrium, potassium, chloride, creatinine, and glucose levels, as well as osmolality, urine osmolality, and $\mathrm{pH}$ were measured. Other laboratory findings, such as full blood count and $\mathrm{N}$-terminal pro-B-type natriuretic peptide (NT-proBNP) plasma concentrations were collected in most patients prior to randomization.

\subsection{Outcome Measurements}

Clinical assessment included daily measurement of patient body mass, arterial pressure, and heart rate. Dyspnea was measured using a $10^{\circ}$ visual analog scale and the $5^{\circ}$ Likerttype scale.

Fluid balance was estimated on the basis of daily urine collection, carried out over $24 \mathrm{~h}$, from 6:00am to 6:00am the following day. The balance of intake fluids was 
Fig. 1 Study protocol. Acetazolamide was administered in 10 of the 20 study patients

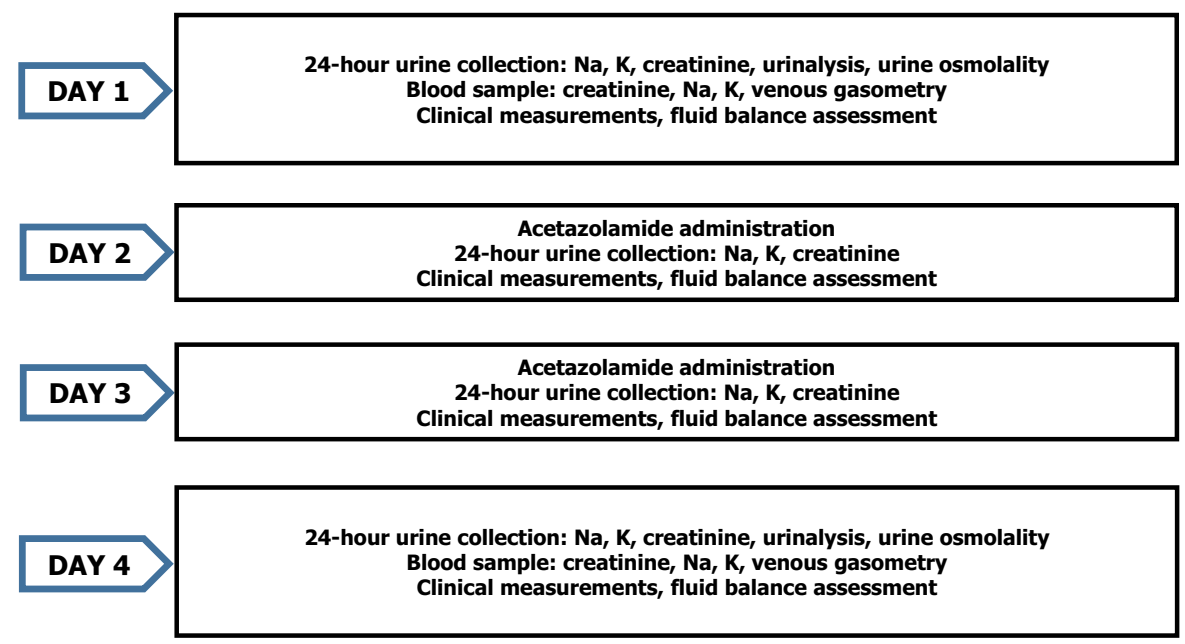

All patients received loop diuretics and other treatments according to the treating physicians' decisions monitored using a questionnaire completed by patients on an ongoing basis. Additionally, the amount of intake fluids was controlled on an ongoing basis by the auxiliary staff in the ward.

Study endpoints included diuresis and fluid balance on each consecutive day, mean diuresis and fluid balance on days 2 and 3 and days 2, 3 and 4, difference in diuresis, and fluid balance on day 1 versus all other days.

\subsection{Statistical Analysis}

The results are presented as mean \pm 1 standard deviation (SD) or median with interquartile range and 95\% confidence interval. Variables were compared using Student's $t$ test or the Mann-Whitney $U$ test as the groups were too small to adequately assess the eventual normality. Statistical significance was set at a probability level of $<0.05$.

The statistical packet Statistica version 10 (data analysis software system; StatSoft, Inc.) was employed for the purpose of calculations (2011).

\section{Results}

\subsection{Study Population}

Twenty patients were included in the study, 10 of whom were randomized to acetazolamide and 10 to the control. The mean age of patients was $72 \pm 12$ years, $85 \%$ were men, and mean left ventricle $\mathrm{EF}$ was $33.8 \pm 11.4 \%$. Ninety-five percent of patients received furosemide $(80 \%$ intravenously), $30 \%$ received oral torasemide (some patients received both furosemide and torasemide), $50 \%$ received spironolactone ( $30 \%$ intravenously), $10 \%$ received oral eplerenone, and $10 \%$ received oral hydrochlorothiazide. Mean intravenous furosemide dose was $101 \mathrm{mg} /$ day in the acetazolamide group and $108 \mathrm{mg} /$ day in the notreatment group. With adjustment for a diuretic potency (oral furosemide twofold lower potency and oral torasemide twofold higher potency than intravenous furosemide), the mean initial dose of loop diuretics converted to intravenous furosemide was $90 \mathrm{mg} /$ day in the acetazolamide group and $122 \mathrm{mg} /$ day in the control group [8]. The difference was not statistically significant. The mean dose of loop diuretics in the following days was similar in both groups (no statistically significant differences).

The full demographics and clinical characteristics of both study groups are presented in Table 1 . The mean adjusted dose of in vitro loop diuretics are presented in Table 2.

\subsection{Diuresis}

Data on mean diuresis and natriuresis on each consecutive day are presented in Table 3. There was no statistically significant difference between values for diuresis and natriuresis in the acetazolamide and control groups according to Student's $t$ test and the Mann-Whitney $U$ test. However, there was a significant increase in mean urine $\mathrm{pH}$ on day 4 versus day 1 in the acetazolamide group versus the control group $(+1.07$ vs. $-0.3 ; p=0.02)$. Urine alkalization confirmed the lasting effect of acetazolamide treatment on day 4.

\subsection{Fluid Balance}

A comparison of fluid balance between the groups is presented in Table 4. There was a trend towards a negative 
Table 1 Baseline demographics and clinical characteristics in the control and acetazolamide-treated groups

\begin{tabular}{|c|c|c|c|}
\hline Variable & Acetazolamide $[n=10]$ & Control $[n=10]$ & $p$ value \\
\hline Age, years [mean $( \pm \mathrm{SD})]$ & $73.0(8.6)$ & $71.2(14.4)$ & 0.74 \\
\hline Men & $8(80)$ & $9(90)$ & 0.56 \\
\hline Charlson comorbidity index (sum) [median] & 3 & 3 & 0.34 \\
\hline Ejection fraction, $\%$ [mean $( \pm \mathrm{SD})]$ & $37.5(13.0)$ & $30.2(8.7)$ & 0.16 \\
\hline Heart failure etiology & & & 0.41 \\
\hline Ischemic & $6(60)$ & $7(70)$ & \\
\hline Other & $4(40)$ & $3(30)$ & \\
\hline Baseline NYHA class & & & 0.14 \\
\hline II & $4(40)$ & $1(10)$ & \\
\hline III & $6(60)$ & $9(90)$ & \\
\hline Coronary artery disease & $8(80)$ & $7(70)$ & 0.63 \\
\hline Arterial hypertension & $8(80)$ & $8(80)$ & 1.0 \\
\hline Atrial fibrillation & $8(80)$ & $6(60)$ & 0.36 \\
\hline Chronic lung disease & $2(20)$ & $2(20)$ & 1.0 \\
\hline Chronic kidney disease & $6(60)$ & $6(60)$ & 1.0 \\
\hline Depression & $1(10)$ & $2(20)$ & 0.56 \\
\hline Diabetes mellitus & $7(70)$ & $5(50)$ & 0.39 \\
\hline \multicolumn{4}{|l|}{ Pharmacotherapy during study } \\
\hline ACE inhibitor/ARB & $8(80)$ & $8(80)$ & 1.0 \\
\hline Aspirin & $5(50)$ & $6(60)$ & 0.67 \\
\hline Statin & $8(80)$ & $5(50)$ & 0.18 \\
\hline$\beta$-blocker & $9(90)$ & $10(100)$ & 0.33 \\
\hline Antibiotics & $5(50)$ & $7(70)$ & 0.39 \\
\hline Amiodarone & $7(70)$ & $5(50)$ & 0.39 \\
\hline OAC/LMWH & $7(70)$ & $8(80)$ & 0.063 \\
\hline Proton pump inhibitor & $7(70)$ & $9(90)$ & 0.29 \\
\hline White blood cells, $\mathrm{K} / \mu \mathrm{L}$ [mean $( \pm \mathrm{SD})]$ & $8.95(3.18)$ & $7.70(1.60)$ & 0.28 \\
\hline Hemoglobin, g/dL [mean $( \pm$ SD)] & $12.19(2.64)$ & $12.39(1.78)$ & 0.85 \\
\hline Troponin T, ng/L [mean $( \pm \mathrm{SD})]$ & $43.73(46.39)[n=9]$ & $65.63(34.40)[n=7]$ & 0.31 \\
\hline $\mathrm{CRP}, \mathrm{mg} / \mathrm{dL}[$ mean $( \pm \mathrm{SD})]$ & $35.77(63.52)[n=9]$ & $67.51(107.39)[n=9]$ & 0.46 \\
\hline Natrium, mEq/L [mean $( \pm \mathrm{SD})]$ & $138.60(3.37)$ & $137.40(4.79)$ & 0.53 \\
\hline Potassium, mEq/L [mean $( \pm \mathrm{SD})]$ & $4.48(0.53)$ & $4.97(0.86)$ & 0.14 \\
\hline Creatinine, $\mu \mathrm{mol} / \mathrm{L}[$ mean $( \pm \mathrm{SD})]$ & $137.05(42.44)$ & $141.47(76.93)$ & 0.88 \\
\hline NT-proBNP, pg/mL [mean $( \pm$ SD)] & $7241.29(5417.86)[n=7]$ & $8704.44(5966.80)[n=9]$ & 0.62 \\
\hline
\end{tabular}

Data are expressed as $n(\%)$ unless otherwise specified

$A C E$ angiotensin-converting enzyme, $A R B$ angiotensin receptor blockers, $C R P$ C-reactive protein, $L M W H$ low molecular weight heparin, $N T$ proBNP N-terminal pro-B-type natriuretic peptide, NYHA New York Heart Association, $O A C$ oral anticoagulants, $S D$ standard deviation

fluid balance in the acetazolamide group on days 3 and 4 , whereas no such trend was observed in the control group. The difference on day 4 fluid balance and mean days 3 and 4 fluid balance between the acetazolamide and control groups was statistically significant, assuming a normal variable distribution (Student's $t$ test), whereas the Mann-Whitney $U$ test yielded no statistical significance.

\subsection{Dyspnea Measurement}

According to both scales, dyspnea was less pronounced in the acetazolamide group (Table 5). The difference escalated over each subsequent day, reaching statistical significance (Student's $t$ test) on days 2, 3 and 4, but the findings were not significant when the Mann-Whitney $U$ test was used. 
Table 2 Mean \pm SD dose of loop diuretics (doses converted to intravenous furosemide dose)
Table 3 Comparison of diuresis and natriuresis between the control and acetazolamidetreated groups

\begin{tabular}{lclc}
\hline Day of the study & Acetazolamide $(n=10)$ & Control $(n=10)$ & $p$ value $^{\mathrm{a}}$ \\
\hline Day 1 & $90 \pm 51$ & $122 \pm 59$ & 0.42 \\
Day 2 & $118 \pm 78$ & $130 \pm 65$ & 0.28 \\
Day 3 & $103 \pm 64$ & $142 \pm 80$ & 0.95 \\
Day 4 & $110 \pm 73$ & $152 \pm 97$ & 0.16 \\
\hline
\end{tabular}

Adjustment for a diuretic potency: oral furosemide has a twofold lower potency and oral torasemide a twofold higher potency than intravenous furosemide [8]

$S D$ standard deviation

a $p<0.05$ for Student's $t$ test considered statistically significant

\begin{tabular}{llll}
\hline Variable & Acetazolamide $(n=10)$ & Control $(n=10)$ & $p$ value $^{\mathrm{a}}$ \\
\hline Diuresis, mL [mean ( \pm SD)] & & & \\
$\quad$ Day 1 & $2351.0(806.8)$ & $2614.00(621.3)$ & 0.42 \\
Day 2 & $2272.0(707.1)$ & $2802.00(1311.8)$ & 0.28 \\
Day 3 & $2641.0(568.1)$ & $2617.00(1172.3)$ & 0.95 \\
Day 4 & $2898.0(1160.9)$ & $2252.00(800.5)$ & 0.16 \\
Mean diuresis, days 3 and 4 & $2769.5(800.6)$ & $2434.50(951.3)$ & 0.41 \\
Mean diuresis, days 2, 3 and 4 & $2603.6(629.1)$ & $2557.00(1065.4)$ & 0.91 \\
Natriuresis, mmol/L [mean ( \pm SD)] & & & \\
Day 1 & $207.9(91.6)$ & $260.88(149.6)$ & 0.40 \\
Day 2 & $212.9(104.2)$ & $247.11(138.7)$ & 0.55 \\
Day 3 & $253.1(72.6)$ & $240.70(131.5)$ & 0.81 \\
Day 4 & $258(129.9)$ & $213.3(112.3)$ & 0.43 \\
\hline
\end{tabular}

$S D$ standard deviation

${ }^{\mathrm{a}} p<0.05$ for Student's $t$ test considered statistically significant

\begin{tabular}{llrl}
\hline Fluid balance, $\mathrm{mL}[\mathrm{mean}( \pm \mathrm{SD})]$ & Acetazolamide $(n=10)$ & Control $(n=10)$ & $p$ value $^{\mathrm{a}}$ \\
\hline Day 1 & $-172.0(968.7)$ & $-165.0(858.9)$ & 0.99 \\
Day 2 & $168.0(1052.1)$ & $-198.0(812.9)$ & 0.40 \\
Day 3 & $-416.0(486.5)$ & $152.0(735.2)$ & 0.057 \\
Day 4 & $-666.0(1194.4)$ & $332.0(704.7)$ & 0.035 \\
Fluid balance, days 3 and 4 & $-541.0(774.3)$ & $242.0(655.9)$ & 0.025 \\
Fluid balance, days 2, 3 and 4 & $-304.7(596.0)$ & $95.3(681.6)$ & 0.18 \\
\hline
\end{tabular}

$S D$ standard deviation

${ }^{\mathrm{a}} p<0.05$ for Student's $t$ test considered statistically significant

\section{Discussion}

The results of our pilot study confirm the potential effectiveness of acetazolamide in the current era of diuretic therapy in heart failure. To our knowledge, only two other human studies have been published over the past 30 years with the use of acetazolamide as a diuretic agent in heart failure [7, 9]. In a small prospective study in nine patients with congestive heart failure, Knauf and Mutschler showed that adding $250 \mathrm{mg}$ of acetazolamide to $40 \mathrm{mg}$ of furosemide produced a similar increase in the rate of diuresis as that achieved by doubling the dose of furosemide [9]. Furthermore, Verbrugge et al. conducted a single-center, prospective cohort study in 54 patients with decompensated heart failure, in which a strict scheme of loop diuretic dose (intravenous bumetanide) was required per protocol. Physicians were encouraged to use combination diuretic therapy: a thiazide diuretic was recommended in patients with kidney dysfunction (estimated glomerular filtration rate $<40 \mathrm{~mL} / \mathrm{min}$ ); the addition of 
Table 5 Comparison of dyspnea scores between the control and acetazolamide-treated groups

\begin{tabular}{lccl}
\hline Variable & Acetazolamide $(n=10)$ & Control $(n=10)$ & $p$ value $^{\mathrm{a}}$ \\
\hline \multicolumn{2}{l}{ Visual dyspnea scale ${ }^{\mathrm{b}}[$ mean $( \pm \mathrm{SD})]$} & & \\
Day 1 & $26(27)$ & $42(13)$ & 0.12 \\
Day 2 & $18(18)$ & $41(16)$ & 0.014 \\
Day 3 & $16(21)$ & $38(19)$ & 0.038 \\
Day 4 & $9(9)$ & $35(16)$ & 0.0006 \\
Likert [mean $( \pm \mathrm{SD})]$ & & \\
Day 1 & $2.278(1.1)$ & $3(0.8)$ & 0.13 \\
Day 2 & $1.722(0.7)$ & $2.833(0.8)$ & 0.0076 \\
Day 3 & $1.667(0.97)$ & $2.778(1.4)$ & 0.07 \\
Day 4 & $1.444(0.64)$ & $2.222(0.83)$ & 0.041 \\
\hline
\end{tabular}

$S D$ standard deviation

a $p<0.05$ for Student's $t$ test considered statistically significant

${ }^{\mathrm{b}}$ Presented in $\mathrm{mm}(0-100 \mathrm{~mm})$

acetazolamide was recommended when the serum urea to creatinine ratio was $>50$; and spironolactone was recommended in patients without hyperkalemia. Overall, $69 \%$ of patients received different types of combination diuretic therapy. The addition of acetazolamide was one of the few statistically significant factors that increased natriuresis (the other being higher diastolic blood pressure and lower plasma aldosterone) [7].

Several mechanisms may explain the potential effectiveness of acetazolamide in combination diuretic therapy. Sodium reabsorption in proximal tubules may be increased in patients receiving chronic loop diuretic therapy or with decompensated heart failure, which in turn weakens the effectiveness of loop diuretics. This type of diuretic resistance may not be effectively treated by combination therapy with distally acting diuretics such as thiazides or mineralocorticoid receptor antagonists; however, the use of acetazolamide, a proximal tubule-acting diuretic, may be effective [9]. Anhydrase inhibitors may also enhance the diuretic effect of thiazides. As shown in animal models, lower urine $\mathrm{pH}$ may upregulate the distal tubule sodium chloride co-transporter, despite the administration of thiazide [9]. Thus, in theory and in some animal models, alkalization of the urine may augment the usefulness of thiazide diuretics [10].

There are several limitations of our study. At the time the study was designed, in 2015, we were using the 2012 ESC heart failure guidelines [11]. These guidelines defined normal $\mathrm{EF}$ as $>50 \%$, whereas patients with $\mathrm{EF}$ in the range of $35-50 \%$ were defined as in the 'grey zone'. We decided to include patients with clearly reduced EF $(<35 \%)$ as well as those with 'grey zone' EF (35-50\%). According to current guidelines, we included patients with reduced $(<40 \%)$ and mid-range EF $(40-49 \%)$ [3]. The mean EF in our group was $33.8 \pm 11.3 \%$.

The small size of our study, as well as the lack of blinding, imply the need for exercising caution in interpreting our results. Many potential confounding factors influence diuresis, such as variability in ambient and body temperature and humidity, which may be difficult to avoid even in a clinical trial. The wide SDs in our results indicate a high probability of chance findings as an explanation of our results. However, the limited clinical experience mentioned above encourages generation of a hypothesis that acetazolamide may augment the diuretic response to other diuretic agents.

\section{Conclusions}

The results of this pilot study suggest that adding acetazolamide to other stable diuretic drugs in patients hospitalized with heart failure exacerbation may produce an additional diuretic effect and alleviation of dyspnea. These findings need to be confirmed in a larger randomized controlled study.

Acknowledgements Editorial support was provided by Sophie Rushton-Smith, PhD (MedLink Healthcare Communications Limited) and was funded by the authors.

\section{Compliance with ethical standards}

Funding This study was conducted thanks to a Centre of Postgraduate Medical Education Grant (Number 501-1-10-14-16). The study drug was provided free of charge by the Polish manufacturer (Polpharma).

Conflict of interest Tomasz Imiela and Andrzej Budaj have no conflicts of interest to declare.

Ethics approval This study was approved by the Postgraduate Medical School Ethics Committee (Approval No. 17/PB/2015) and all procedures in the study were in accordance with the 1964 Declaration of Helsinki.

Informed consent Written informed consent was obtained from all patients.

Open Access This article is distributed under the terms of the Creative Commons Attribution-NonCommercial 4.0 International License (http://creativecommons.org/licenses/by-nc/4.0/), which permits any noncommercial use, distribution, and reproduction in any medium, provided you give appropriate credit to the original author(s) and the source, provide a link to the Creative Commons license, and indicate if changes were made.

\section{References}

1. Mosterd A, Hoes AW. Clinical epidemiology of heart failure. Heart. 2007;93(9):1137-46. 
2. Kelder JC, Cramer MJ, van Wijngaarden J, van Tooren R, Mosterd A, Moons KG, et al. The diagnostic value of physical examination and additional testing in primary care patients with suspected heart failure. Circulation. 2011;124(25):2865-73.

3. Ponikowski P, Voors AA, Anker SD, Bueno H, Cleland JG, Coats AJ, et al. 2016 ESC Guidelines for the diagnosis and treatment of acute and chronic heart failure: The Task Force for the diagnosis and treatment of acute and chronic heart failure of the European Society of Cardiology (ESC)Developed with the special contribution of the Heart Failure Association (HFA) of the ESC. Eur Heart J. 2016;37(27):2129-200.

4. Kassamali R, Sica DA. Acetazolamide: a forgotten diuretic agent. Cardiol Rev. 2011;19(6):276-8.

5. Hanley T, Platts MM. Acetazolamide (diamox) in the treatment of congestive heart-failure. Lancet. 1956;270(6919):357-9.

6. Neuberg GW, Miller AB, O'Connor CM, Belkin RN, Carson PE, Cropp AB, et al. Diuretic resistance predicts mortality in patients with advanced heart failure. Am Heart J. 2002;144(1):31-8.

7. Verbrugge FH, Dupont M, Bertrand PB, Nijst P, Penders J, Dens $\mathrm{J}$, et al. Determinants and impact of the natriuretic response to diuretic therapy in heart failure with reduced ejection fraction and volume overload. Acta Cardiol. 2015;70(3):265-73.

8. Felker GM, Mentz RJ. Diuretics and ultrafiltration in acute decompensated heart failure. J Am Coll Cardiol. 2012;59(24):2145-53.

9. Knauf H, Mutschler E. Sequential nephron blockade breaks resistance to diuretics in edematous states. J Cardiovasc Pharmacol. 1997;29(3):367-72.

10. Zahedi K, Barone S, Xu J, Soleimani M. Potentiation of the effect of thiazide derivatives by carbonic anhydrase inhibitors: molecular mechanisms and potential clinical implications. PLoS One. 2013;8(11):e79327.

11. McMurray JJ, Adamopoulos S, Anker SD, Auricchio A, Bohm M, Dickstein K, et al. ESC Guidelines for the diagnosis and treatment of acute and chronic heart failure 2012: the task force for the diagnosis and treatment of acute and chronic heart failure 2012 of the European Society of Cardiology. Developed in collaboration with the Heart Failure Association (HFA) of the ESC. Eur Heart J. 2012;33(14):1787-847. 